\title{
A method to achieve clear-sky data-volume download in satellite links affected by tropospheric attenuation
}

\author{
Emilio Matricciani* ${ }^{\dagger}$ \\ Dipartimento di Elettronica, Informazione e Bioingegneria, Politecnico di Milano, Milan, Italy
}

\begin{abstract}
SUMMARY
For data downloading from satellites, the traditional approach of considering the complementary probability distribution, $P\left(A_{\text {trop }}\right)$ of the total tropospheric attenuation $A_{\text {trop }}(\mathrm{dB})$, at a frequency, polarization, and elevation angle, may be too pessimistic, and it may lead to large overdesign. If the data volume downloaded in a given observation time $T_{o b s}$ (a day, a week, a month, etc.), with a constant probability of symbol error, is more valuable than the instantaneous symbol, or bit rate (as it may be the case in Earth resources observation or in other services that allow long delays in communicating data), another approach can avoid overdesign, namely the various adaptive coding and modulation techniques. We study a particular time integral of $A_{\text {trop }}(t)$ : (i) to define the average efficiency of a radio channel faded by the troposphere and (ii) to design a method that can theoretically achieve the same volume of data downloaded in clear-sky conditions (no troposphere), even if the radio link is faded by the troposphere. The average efficiency and its bounds can be calculated from the complementary probability distribution of $A_{\text {trop }}(t)$. We explicitly apply the theory to radio links faded by rain, by using the experimental rain-attenuation time series measured with satellite ITALSAT in a $37.8^{\circ}$ slant path, at $18.7,39.6$, and $49.5 \mathrm{GHz}$ at Spino d'Adda (Italy), and to those simulated with the synthetic storm technique. Based on the average efficiency, we define the method that can achieve in $T_{o b s}$, theoretically, the same data volume as in clear sky, directly applicable to quadrature phase-shift keying, multiple phase-shift keying, and Shannon capacity theorem. The method requires a fixed increase in power margin, and bandwidth, compared with clear-sky conditions, and delivers an average symbol rate equal to the maximum symbol rate obtainable when $A_{\text {trop }}(t)=0$. The method can also be used in terrestrial links at any frequency. We compare its theoretical performance with the traditional adaptive coding and modulation techniques and show that, even theoretically, these techniques cannot achieve unitary efficiency as, on the contrary, the novel method can do. Copyright @ 2015 John Wiley \& Sons, Ltd.
\end{abstract}

Received 18 December 2014; Revised 23 May 2015; Accepted 17 June 2015

KEY WORDS: $\quad$ satellite link efficiency; tropospheric fading; rain attenuation; data-volume download; ACM

\section{A SINGLE PARAMETER FOR CHARACTERIZING RADIO LINKS AFFECTED BY TROPOSPHERIC FADING}

Real-time satellite communications at frequencies above $10 \mathrm{GHz}$ are characterized with the long-term probability distribution, $P\left(A_{\text {trop }}\right)$, of total tropospheric attenuation $A_{\text {trop }}(\mathrm{dB})$ (a combination of attenuation due to rain, hail, snow, mist, water vapor, clouds, and oxygen), at a frequency, polarization, and elevation angle. This $P\left(A_{\text {trop }}\right)$ refers to an average year, or to a shorter observation period, $T_{\text {obs }}$, for example, a month, and is useful to calculate link budget and to assess link availability. The same approach holds for second-order statistics, such as fade duration and time derivative of fade (rate of change), which describe fading dynamics, especially with regard to rain attenuation, the most rapidly changing and less predictable tropospheric fade in slant paths. Fade duration statistics give information on the duration of continuous outages due to rain, and they set the stage for assessing how different

*Correspondence to: Emilio Matricciani, Dipartimento di Elettronica, Informazione e Bioingegneria, Politecnico di Milano, Milan, Italy. ${ }^{\dagger}$ E-mail: Emilio.Matricciani@polimi.it 
real-time communication services tolerate short or long outages. Rate-of-change statistics give information on the dynamics of rain fade, and they set the stage for designing the methods that, in real time, try to match the instantaneous signal-to-noise ratio, or some equivalent parameter, to the link fade. At higher frequencies, such as $K_{a}$ band, also the fading due to the other troposphere constituents mentioned previously must be considered. Because these latter effects are dynamically slower than rain attenuation, currently they are commonly considered as static.

For data downloading, however, this approach may be too pessimistic, and it may lead to large overdesign. In fact, if the size of the data volume downloaded in a given observation time $T_{o b s}$ (a day, a week, a month, etc.) is more valuable than the instantaneous symbol or bit rate (as it may be the case in Earth resources observation or in other services that allow long delays in communicating data), another approach can be more useful and avoid overdesign, namely the various adaptive coding and modulation (ACM) techniques (in the vast literature on the subject, for brevity, see [1-5]).

This paper investigates an alternative method for performing ACM in satellite links subjected to time-varying tropospheric fading, with quadrature phase-shift keying (QPSK) (or multiple phase-shift keying). Whereas the traditional ACM approaches adjust constellation size and code rate for a fixed symbol rate to maximize the instantaneous bit rate, the approach described here adjusts the symbol rate to optimize the number of symbols and data bits downloaded during $T_{o b s}$ while maintaining a QPSK scheme. However, to be applicable, the method requires to increase the transmitted power and bandwidth. The latter requirement is not intuitive and will be justified in the following.

We first discuss a single parameter for characterizing radio channels faded by the troposphere, namely what we term the average efficiency, and then we use it to propose an ideal method that, theoretically, could achieve the same total amount of downloaded data as if there were no troposphere ("clear sky'). The method may be a potential choice to add to the current ACM techniques. We consider only the theoretical aspects, even if we report some experimental results concerning the numerical values of the average efficiency (to which the method is tightly related), concerning only rain attenuation.

Time (or space) integrals of physical quantities are defined and used in many engineering areas and in science, for example, in physics. For the first time, as far as we know, we study a particular time integral of tropospheric attenuation $A_{\text {trop }}(t)$ and discuss its meaning and possible applications in communication systems in which the total volume of data downloaded in the observation time $T_{o b s}$, with a constant probability of symbol and bit error, is more important than the instantaneous symbol or bit rate. In particular, we study the following integral:

$$
\tau=\int_{T_{\text {obs }}} 10^{-A_{\text {trop }}(t) / 10} d t
$$

Notice that $\tau \leq T_{o b s}$ has the dimension of time and can be used to define the average efficiency of a faded radio channel.

First, we develop the theory that, from (1), defines the average efficiency. Then, we apply the theory to the specific case of rain attenuation $A(t)(\mathrm{dB})$ and calculate this parameter from the experimental rain-attenuation time series measured with the satellite ITALSAT, in a $37.8^{\circ}$ slant path, at 18.7 , 39.6, and $49.5 \mathrm{GHz}$ at Spino d'Adda (Italy), and from those simulated with the synthetic storm technique (SST) [6] in the same locality. In case of rain, in most of the observation time $T_{o b s}$, we will not experiment rain attenuation, of course, and thus, instead of $T_{o b s}$, only the total rain-attenuation time $T_{A}$ must be considered in (1), with $T_{A}<T_{o b s}$.

Second, we apply the concept of average efficiency for defining the ideal clear-sky sizing method mentioned previously, which theoretically requires a fixed increase in power margin and a variable and larger bandwidth and delivers, in $T_{o b s}$ (or $T_{A}$ ), an average symbol rate equal to the maximum symbol rate obtainable when $A_{\text {trop }}(t)=0$ (or $A=0$ ), thus perfectly combating any tropospheric fading.

Section 2 defines the equivalent download time and the average efficiency of radio channels for QPSK and for Shannon capacity theorem. Section 3 reports the experimental results of average efficiency for rain attenuation, obtained with ITALSAT and SST data. Section 4 defines the ideal method and extends (1) to fades other than rain attenuation. Section 5 discusses the theoretical and basic differences between the current ACM techniques and the new method. Section 6 concludes by indicating some open problems and future developments. Appendices A-D report mathematical developments that justify some theoretical results. 


\section{EQUIVALENT DOWNLOAD TIME DURING RAIN ATTENUATION AND CHANNEL AVERAGE EFFICIENCY}

Let us consider the baseband equivalent (ideal) AWGN channel for the QPSK (Appendix A), with onesided equivalent bandwidth $R_{S}(1+\delta) / 2=B_{R F}$, being $R_{S}$ the total symbol rate, $B_{R F}$ the radiofrequency bandwidth, $\delta$ the roll-off factor, and $P_{r}$ the carrier power (sum of the power of the two orthogonal carriers) received in free space and clear-sky conditions (the equivalent power received after channel decoding is given by $P_{r} g_{c}$, where $g_{c}$ is the constant coding gain in natural units). Standard calculations show that the instantaneous symbol rate $R_{s}(t)$ at time $t$, during a rain-attenuation event, is given (Appendix B) by

$$
R_{S}(t) \geq \frac{P_{r} \times 10^{-A_{G} / 10} \times 10^{-A(t) / 10}}{k\left(T_{m}+T_{r e c}\right) \rho}=R_{o} \times 10^{-A(t) / 10}
$$

The bit rate $R_{b}(t)$ is proportional to $R_{s}(t)$ according to the code rate $R_{c}$ applied. Moreover, in (2), $T_{m}(\mathrm{~K})$ is the average physical temperature of rain (assumed to be constant), and $T_{\text {rec }}(\mathrm{K})$ is the receiver equivalent noise temperature; $A_{G}(\mathrm{~dB})$ is the total attenuation due to water vapor, oxygen, and clouds and is supposed to be constant during rain attenuation (we will remove this restriction in Section 4); $\rho=E_{b} / N_{o}$ is the signal-to-noise ratio, $E_{b}$ is the received energy per bit, and $N_{o}=k\left(T_{m}+T_{\text {rec }}\right)$ is the onesided noise power density. The inequality (2) arises because we assume that the antenna noise temperature $T_{s k y} \leq T_{m}(\mathrm{~K})$ is upper bounded by $T_{m}$, a conservative hypothesis that models rain fade, for noise calculation, as due to a passive attenuator at temperature $T=T_{m}$ with $A \rightarrow \infty$ (Appendix B). We do not mention scintillation because, according to the results reported as follows, this phenomenon is negligible for our theory.

Let us study (2) under the hypothesis of providing the minimum equivalent and tolerable $\rho_{o}$ (fixed by the tolerated maximum probability of symbol and bit error) by reducing $R_{s}(t)$. In other words, we suppose that the link can match dynamically and perfectly $R_{S}(t)$ to the slow (compared with the symbol rate) time-varying $A(t)$. The amount of symbols downloaded during the rain-attenuation time $T_{A}$ is given by

$$
D=\int_{T_{A}} R_{S}(t) d t=R_{o} \int_{T_{A}} 10^{-A(t) / 10} d t=R_{o} \tau
$$

As evidenced in (3), the integral (1), applied to rain attenuation now, gives the equivalent time $\tau$ during which the constant rate $R_{o}$ delivers the same total volume of downloaded symbols $D$. In other words, the perfect matching (2) in $T_{A}$ is equivalent to transmitting the theoretical maximum symbol rate $R_{o}$ in an ideal channel with $A(t)=0 \mathrm{~dB}$ but for the shorter equivalent interval $\tau$.

The integral (3) plays the same role for multiple phase-shift keying modulation and for Shannon capacity formula. For Shannon, by adopting natural logarithms, and recalling that $\ln (1+\gamma) \leq \gamma$, we can write

$$
C=B_{R F} \times \ln (1+\gamma) / \ln 2 \leq\left(P / N_{o}\right) / \ln 2
$$

where $\gamma=P /\left(N_{o} B_{R F}\right)$ is the carrier-to-noise ratio and $P=P_{r} \times 10^{-A_{G} / 10} \times 10^{-A(t) / 10}$ is the received power. In (4), let us assume $\ln (1+\gamma) \approx \gamma$, acceptable if $\gamma(t)<1(0 \mathrm{~dB})$, because this is likely most of the range (actually between -3 and $6 \mathrm{~dB}$ ) for adopting QPSK in the current ACM techniques, so that we make consistent comparisons. We obtain

$$
\begin{gathered}
C(t)=\frac{P_{r} \times 10^{-A_{G} / 10}}{k\left(T_{m}+T_{r e c}\right) \ln 2} \times 10^{-A(t) / 10}=C_{o} \times 10^{-A(t) / 10} \\
\Gamma=\int_{T_{A}} C(t) d t=C_{o} \int_{T_{A}} 10^{-A(t) / 10} d t=C_{o} \tau
\end{gathered}
$$

Therefore, we find the same equivalent time of QPSK, an important result because $\tau$ and the efficiency $\tau$, defined as follows, represent, respectively, the least equivalent time and the largest efficiency, because they refer to $C_{o}$.

Now, we can define the average efficiency $\eta$, both for QPSK (2) and for Shannon capacity (5), as the ratio between the number of downloaded symbols and the number of downloadable symbols when 
$A(t)=0$, both in $T_{A}$. Because the latter is given by $R_{o} T_{A}$ (or $C_{o} T_{A}$ ), from (5) or (6), we obtain the following average efficiency:

$$
\eta=\int_{T_{A}} 10^{-A(t) / 10} d t / T_{A}=\tau / T_{A}
$$

Therefore, the total data volume is $D$ (or $\Gamma$ ), and, consequently, the average symbol rate provided to the user, with the required quality, is given by

$$
\begin{aligned}
& \Re=D / T_{A}=\eta R_{o} \\
& \Re=\Gamma / T_{A}=\eta C_{o}
\end{aligned}
$$

In other words, the average symbol rate $\mathfrak{R}$ is a fraction $\eta$ of the maximum rate obtainable when $A(t)=0$, which is in clear-sky conditions. The average efficiency can thus characterize radio links with a single number.

Notice that we do not need to consider rain-attenuation time series in (7) because it corresponds to the average value of the random variable $10^{-A / 10}$, which is, of course, the instantaneous efficiency. We just need to know the conditional complementary probability distribution $P_{c}(A)=P(A) / P_{o}$, where $P_{o}=P(A>0)=T_{A} / T_{o b s}$ is the probability of rain attenuation. Standard calculations give the following final result (Appendix C):

$$
\eta=1-(\ln 10 / 10) \int_{0}^{\infty} 10^{-A / 10} P_{c}(A) d A
$$

From the aforementioned discussion, it is obvious that we do not need to know fade duration statistics to calculate the average efficiency and that we could introduce in (2) further constant fading or gain (site diversity, time diversity, etc.), which will only change $R_{o}$, not the average efficiency (9). This argument applies also to a constant interference power, if we consider it another source of Gaussian noise (worst case according to Shannon). In other words, all constant-power noise sources, or gains, change $R_{o}$ but not $\eta$.

The average efficiency (9) is a random variable limited in a range. Any time we deal with integrals, we can use the Cauchy-Schwarz inequality for finding bounds, given by (Appendix D):

$$
\left[1-\frac{\ln 10}{20} \int_{0}^{\infty} 10^{-A / 20} P_{c}(A) d A\right]^{2} \leq \eta \leq \sqrt{1-\frac{\ln 10}{5} \int_{0}^{\infty} 10^{-A / 5} P_{c}(A) d A}
$$

\section{AVERAGE EFFICIENCY EXPERIMENTAL RESULTS}

We apply the theory to the experimental rain-attenuation time series measured at Spino d'Adda $\left(45.4^{\circ} \mathrm{N}\right.$, $9.5^{\circ} \mathrm{E}, 84 \mathrm{~m}$ a.s.1.), Italy, at $18.7,39.6$, and $49.5 \mathrm{GHz}$, in a $37.8^{\circ}$ slant path (after proper postdetection filtering, the sampling time is $1 \mathrm{~s}$ ), during the ITALSAT experimental campaign, in the years 1993-2000 (7 years). Second, because this data bank in the long term is not statistically complete because some intense rain-attenuation events were missed [7], we apply the SST to a large data bank of rain-rate time series collected at the same site in the years 1993-2002 (10 years), to simulate rainattenuation time series at the same frequencies and elevation angle (Figure 1).

Table I lists the values of the average efficiency at $18.7,39.6$, and $49.5 \mathrm{GHz}$ both for filtered and non-filtered rain-attenuation time series measured with ITALSAT. In the latter, scintillations are present, while in the former, they have been filtered. There is no appreciable difference between the two sets, so that scintillation plays no role in this assessment, at least for the electrically large antenna (offset parabolic antenna of $3.5 \mathrm{~m}$ diameter) used in Spino d'Adda. Because, in the long term, the set of rain-attenuation time series simulated with the SST is more complete than the ITALSAT measurements [7], we base our main analysis and results on these simulated data, also reported in Table I. 


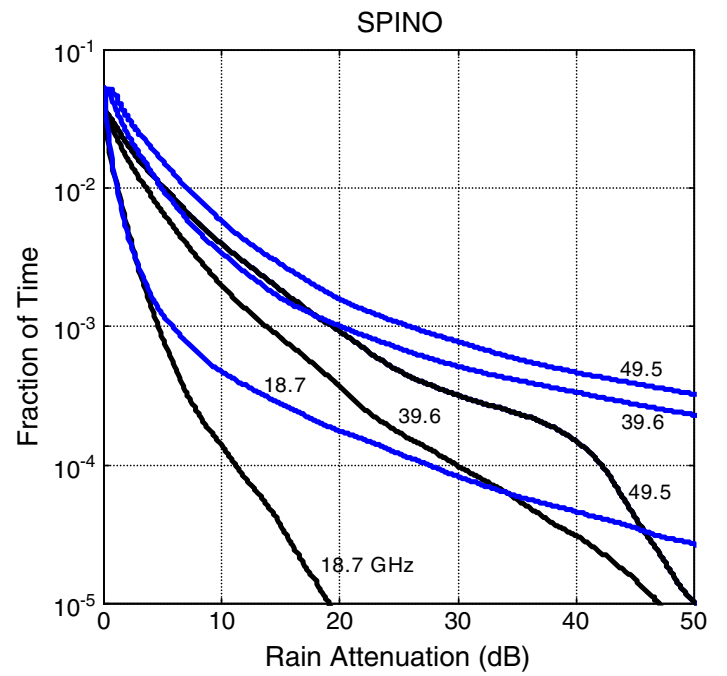

(a)

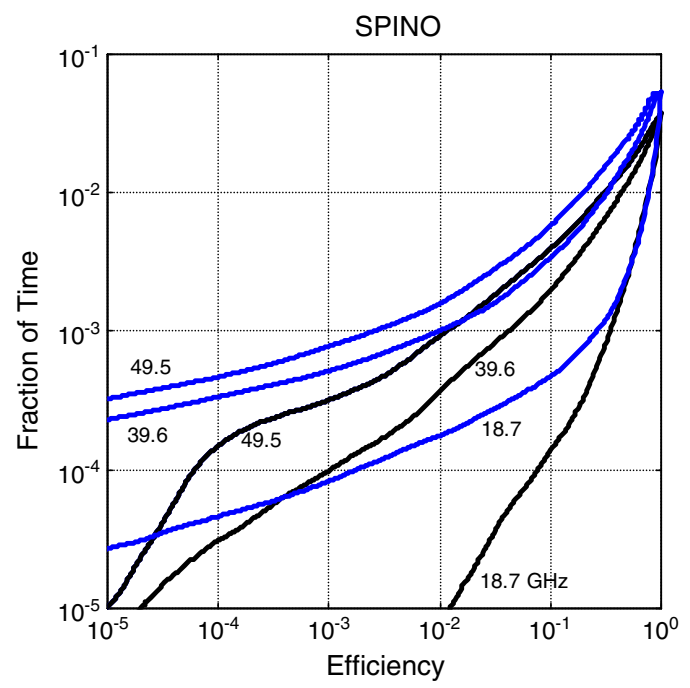

(b)

Figure 1. (a) Complementary probability distribution functions (or fraction of time), $P(A)$, in an average year, of the rain attenuation, $A(\mathrm{~dB})$ in abscissa is exceeded at $18.7,39.6$, and $49.5 \mathrm{GHz}, 37.8^{\circ}$ elevation angle; (b) distribution function (or fraction of time) that the instantaneous efficiency in abscissa is not exceeded in an average year. Black lines represent ITALSAT experimental results, and blue lines denote synthetic storm technique experimental results (Spino d'Adda).

Table I. Average, minimum, and maximum of the average efficiency, in an average year, at 18.7, 39.6, and $49.5 \mathrm{GHz}$, measured from non-filtered (with scintillations) and filtered (no scintillations) ITALSAT rainattenuation time series, in a $37.8^{\circ}$ slant path in Spino d'Adda, 1993-2000.

\begin{tabular}{|c|c|c|c|c|c|c|}
\hline \multirow{2}{*}{ Average efficiency } & \multicolumn{2}{|c|}{$18.7 \mathrm{GHz}$} & \multicolumn{2}{|c|}{$39.6 \mathrm{GHz}$} & \multicolumn{2}{|c|}{$49.5 \mathrm{GHz}$} \\
\hline & Non-filtered & Filtered & Non-filtered & Filtered & Non-filtered & Filtered \\
\hline \multirow[t]{2}{*}{ Minimum } & 0.814 & 0.815 & 0.575 & 0.574 & 0.476 & 0.475 \\
\hline & & 0.845 & & 0.546 & & 0.437 \\
\hline \multirow[t]{2}{*}{ Average } & 0.828 & 0.828 & 0.622 & 0.621 & 0.537 & 0.536 \\
\hline & & 0.859 & & 0.594 & & 0.493 \\
\hline \multirow[t]{2}{*}{ Maximum } & 0.847 & 0.847 & 0.681 & 0.679 & 0.613 & 0.612 \\
\hline & & 0.876 & & 0.650 & & 0.561 \\
\hline
\end{tabular}

Italics give the synthetic storm technique-derived average efficiency in the years 1993-2002. 


\section{A METHOD FOR DOWNLOADING CLEAR-SKY DATA-VOLUME DESPITE FADING}

The concept of average efficiency suggests an ideal method for achieving the same volume of data downloadable in clear-sky conditions. If this method could be perfectly applied, with any fade, the average symbol rate in $T_{o b s}$ would be equal to the maximum symbol rate obtainable when $A_{\text {trop }}(t)=0$, therefore restoring the average efficiency to unity, both for the symbol rate $R_{s}(t)$ and bit rate $R_{b}(t)$. As we show next, the cost to pay for this result is to increase the transmitted power and the bandwidth.

Let us consider rain attenuation explicitly and introduce in (2) a fixed power margin $1 / \eta$, so that we receive the following power:

$$
P_{r, \eta}=\frac{P_{r}}{\eta}
$$

This power does maintain a constant $\rho_{o}$, and thus the tolerated maximum probability of symbol error, only if we adopt the following instantaneous symbol rate:

$$
R_{s}^{*}(t)=\left(R_{o} / \eta\right) \times 10^{-A(t) / 10}
$$

Accordingly, the maximum bandwidth is multiplied by $1 / \eta$ (Appendix A). Therefore, the average symbol rate becomes $\left(R_{o} \tau\right) /\left(\eta T_{A}\right)=R_{o}$.

In other words, as soon as $A(t)=0^{+}$, power and symbol rate are both simultaneously increased (multiplied by $1 / \eta$, a non-intuitive fact), and, during the rain-attenuation event, the transmitter power is maintained constant, while the symbol rate is reduced according to (12). The variation can be large, but in a practical system, we could fix a minimum rate, for example, $\left(R_{o} / \eta\right) / 100$, hence $A(t) \leq 20 \mathrm{~dB}$ in (12), without affecting the value of $\eta$ calculated numerically with (9), because the highest attenuations give a little contribution to the numerical value of (1); see Figure 1.

Now, whichever is the observation time $T_{o b s}$, or $T_{A}$, the average symbol rate would always be equal to $R_{o}$, that is, that obtainable when $A_{\text {trop }}(t)=A(t)=0$. For instance, at Spino d'Adda, at $49.5 \mathrm{GHz}$ (Table I, SST), for the minimum average efficiency (worst case), we need a fixed power margin of $-10 \times \log _{10} \eta=-10 \times \log _{10} 0.437=3.6 \mathrm{~dB}$ and should multiply the bandwidth, at most, by the factor $1 / 0.437=2.3$, during a rain-attenuation event. These considerations apply also to shorter observation times, such as quarters or single months. Table II shows the average efficiency obtained at $49.5 \mathrm{GHz}$ (SST) for each quarter of a calendar year. The worst quarter is the summer one (Table III; July, August, and September, the most rainy months in the area considered), and the worst month in this quarter is September (Table III), when the minimum average efficiency is 0.337 , so that, in this case, we need to increase the power by $4.72 \mathrm{~dB}$ and multiply the bandwidth by 3.0.

Notice that the same procedure can be applied to smaller observation periods, even to a particular day of the year, once data are enough to obtain reliable statistics for calculating or measuring the average efficiency from the long-term $P(A)$ of that day. Notice, however, that because the average efficiency is calculated for the expected (ergodic) average $P(A)$ in $T_{o b s}$, variations around this $P(A)$ in $T_{o b s}$ will cause, of course, variations in the average efficiency, other than those in (10).

The average efficiency does not increase much with frequency. At $100 \mathrm{GHz}$, a frequency at which rain attenuation is maximum, in September the minimum average efficiency is 0.167 , and it becomes 0.176 at $200 \mathrm{GHz}, 0.189$ at $300 \mathrm{GHz}$, and 0.196 at $400 \mathrm{GHz}$ (terahertz band). At $100 \mathrm{GHz}$, the power should be increased by $7.8 \mathrm{~dB}$ and the bandwidth multiplied by $10^{7.8 / 10}=6.0$. These results can make this method useful also for short-length (hundreds of meters) terrestrial line-of-sight radio links at terahertz frequencies. The numerical values of $\eta$ should be assessed once measured or reliable predictions are possible at these higher frequencies, for all types of hydrometeors and oxygen [8].

Table II. Average, minimum, and maximum average efficiencies at $49.5 \mathrm{GHz}$ in a $37.8^{\circ}$ slant path in Spino d'Adda obtained with the synthetic storm technique in the years 1993-2002, for each quarter of a calendar year.

\begin{tabular}{lcccc}
\hline Average efficiency & I & II & III & IV \\
\hline Minimum & 0.488 & 0.447 & 0.354 & 0.428 \\
Average & 0.528 & 0.503 & 0.436 & 0.483 \\
Maximum & 0.582 & 0.570 & 0.528 & 0.552 \\
\hline
\end{tabular}


Table III. Average, minimum, and maximum average efficiencies at $49.5 \mathrm{GHz}$ in a $37.8^{\circ}$ slant path in Spino d'Adda obtained with the synthetic storm technique in the years 1993-2002, for July, August, and September.

\begin{tabular}{lccc}
\hline Average efficiency & July & August & September \\
\hline Minimum & 0.409 & 0.348 & 0.337 \\
Average & 0.490 & 0.436 & 0.416 \\
Maximum & 0.575 & 0.532 & 0.507 \\
\hline
\end{tabular}

The extension of the results to the general case of total tropospheric fading is straightforward. It suffices to adopt, in the foregoing equations, the probability distribution of total fade $P\left(A_{\text {trop }}\right)$ instead of that of rain attenuation $P(A)$. In other words, the theory is general.

\section{THEORETICAL COMPARISON WITH CURRENT ADAPTIVE CODING AND MODULATION TECHNIQUES}

Let us compare, theoretically, the method here proposed with the current ACM techniques by considering first power and bandwidth and then a fundamental limit of current ACM techniques that the novel method has not.

\subsection{Power and bandwidth}

The current ACM techniques attempt to maximize the instantaneous bit rate $R_{b}(t)$, not the total download, as the novel method does, by changing the modulation order $k_{m}$ and/or the code rate $R_{c}$, compatible with $\gamma(t)$ and $\rho_{o}$, while keeping constant both the symbol rate and the transmitter power adopted in clearsky conditions (unless another fade countermeasure, such as adaptive power control, is used, in which case, however, outages are always possible), according to the general relationship $R_{b}(t)=R_{s} k_{m}(t) R_{c}(t)$. This design produces complex modulation/coding schemes because of the frequent switchover of modulation and code rate $[3,4]$.

The novel method requires, as the current ACM techniques, a fixed transmitter power, but larger than that adopted in clear-sky conditions, and, differently than traditional ACM, a variable symbol rate (which can incorporate a variable symbol rate due to the source of information or coding, besides that due to $A(t)$ ), which translates, proportionally, into a variable symbol rate for QPSK. However, the implementation of this method (once $A(t)$ is estimated, an issue also for ACM) requires to change the satellite bandwidth dynamically, and this technology can be demanding and complex.

In other words, while ACM tries to maximize $R_{b}(t)$ by changing the product $k_{\mathrm{m}}(t) R_{\mathrm{c}}(t)$ according to $\gamma(t)$ and $\rho_{o}$, the method proposed here tries to match $R_{s}(t)$ to $A(t)$, according to (12), an action that together with the increased transmitted power (11) restores the average efficiency to unity for QPSK and, more generally, for Shannon capacity, in a given observation period (e.g., a day and a week).

This result is not even theoretically possible with ACM techniques, as we show next, and, therefore, it can motivate further in-depth analysis of the practical applications of the method proposed here.

\subsection{Theoretical limitation of current adaptive coding and modulation techniques}

The modulation that ACM can adopt at constant power and symbol rate to maintain $\rho(t) \geq \rho_{o}$ for the lowest $\gamma(t)$ available is, of course, QPSK. In this case, from (2), the minimum bit rate is given by

$$
R_{b}(t)=R_{o} \times 10^{\left[G_{c}(t)-A(t)\right] / 10} \times R_{c}(t)
$$

where $G_{c}(t)=10 \times \log _{10} g_{c}(t)$ is the time-varying coding gain (dB) (assuming a perfect matching).

Let us assume the most favorable value $R_{c}(t)=1$ for the traditional ACM, so that the average efficiency (9) is now given by

$$
\eta_{A C M}=\int_{T_{A}} 10^{\left[G_{c}(t)-A(t)\right] / 10} d t / T_{A}
$$

Because $G_{c}(t)$ is limited by Shannon, with a practical maximum value around $10 \mathrm{~dB}$, all these ACM techniques cannot always set $G_{c}(t)-A(t)=0$; therefore, $\eta_{A C M}<1$. With the traditional ACM 
techniques, long intervals with $G_{c}(t)<A(t)$ can be frequent (because of fade durations), especially at high frequencies, such as $K_{a}$ band and above, giving long intervals of no service. Theoretically, this cannot happen with the method discussed in this paper.

\section{CONCLUDING REMARKS}

The novel fixed-modulation/adaptive-bit-rate method, described in this paper, is different from the methods used by traditional ACM techniques [1-5]. The novel method can theoretically restore the channel average efficiency to unity, in a given observation interval $T_{o b s}$, for any frequency band, a result that current ACM techniques cannot obtain, even theoretically. Therefore, it may provide another possible ACM adaptive technique, or it can suggest another way of controlling or improving the current ACM techniques. In theory, the method can also be applied to other slow fading mechanisms in clear-sky conditions (multipath) or to time-varying fades due to the troposphere or to interference. This approach could also provide a practical standard reference method to characterize the relative practical benefits of different adaptive countermeasure methods by comparing their efficiencies.

The power margins founded in Section 4 for the specific case of rain attenuation only are realistic and of the same order as the power margins encountered in satellite systems today. The required bandwidth expansion, however, can be large and may pose serious challenges for spectrum regulation. Notice, however, that this variable bandwidth is required, of course, only when the link is mainly faded by rain (which is, of course, the fastest and less predictable fading mechanism of the troposphere), not all the time. Therefore, the same channel could be used by other links faded by rain. In other words, a possible method to provide a variable bandwidth channel to a link faded by rain is by resorting to a spare channel (a shared resource) designed for this purpose. See [9] for the kind of information and detailed analysis necessary in a similar investigation and [5] for a practical use. This channel will be assigned dynamically and momentarily to the link, either downlink or uplink. It should not be an insurmountable difficulty, for example, in Earth observation downlinks where there are only few ground receiving stations and the download takes place only for few minutes at each pass of the satellite (once the corresponding $P(A)$ or $P_{\text {trop }}$ $\left(A_{\text {trop }}\right)$ and average efficiency are known, for this variable elevation-angle link). Of course, this design will add a large complexity to the system because all interested links must be monitored simultaneously, and, in case of many stations interested, the number of shared channels must be designed by considering the probability of requiring the shared resource by several links at the same time.

\section{APPENDIX A. BASEBAND EQUIVALENT AWGN CHANNEL FOR THE QPSK}

Let us refer to one of the two orthogonal carriers of QPSK. It carries $R_{S} / 2$ symbols per second, and it needs a radiofrequency bandwidth $B_{R F}=2 \times R_{S}(1+\delta) / 4=R_{S}(1+\delta) / 2$, which is the base bandwidth occupied by a direct transmission of the full symbol rate $R_{s}$. Because the other orthogonal bandwidth is superposed, QPSK needs the same bandwidth of a direct transmission. Now, for the signal-to-noise ratio, each carrier uses half of the total power transmitted for QPSK, or for the full symbol rate at baseband, but it carries half of the total symbol rate, so that, as the noise power density is the same for both carriers and equal to that found at baseband in the direct transmission, the carrier signal-to-noise ratio is equal to that found with the full rate transmission $R_{s}$ at baseband. The same is valid for the other carrier. In conclusion, the baseband transmission provides the same total symbol rate and probability of symbol error using the same bandwidth and power of QPSK, so that, in ideal conditions (no interference between the two orthogonal carriers), it is the equivalent circuit of QPSK.

\section{APPENDIX B. NOISE CALCULATION}

The system (total) noise is given by $T_{s}=T_{s k y}+T_{\text {rec }}(\mathrm{K})$ where $T_{\text {rec }}(\mathrm{K})$ is the receiver equivalent noise temperature and $T_{s k y}(\mathrm{~K})$ is the antenna noise temperature. $T_{s k y}$ is mainly caused by absorption, not by scattering phenomena. Raindrops, however, absorb and scatter electromagnetic waves, so that rain attenuation is caused by both absorption and scattering. To be conservative, however, we assume that 
$T_{\text {sky }}$ is caused by the total rain attenuation $A(\mathrm{~dB})$, so that, by modeling rain attenuation as caused by an ideal passive medium with average physical temperature $T_{m}(\mathrm{~K}), T_{s k y}$ is given by

$$
T_{s k y}=\frac{10^{A / 10}-1}{10^{A / 10}} T_{m}+\frac{1}{10^{A / 10}} T_{d s}
$$

where $T_{d s}(\mathrm{~K})$ is the space background noise. This is the standard model for $T_{s k y}$, with $T_{m} \approx 275 \mathrm{~K}$ usually assumed in communication link budgets (see [10] for a model of $T_{s k y}$ versus $A$, applicable before saturation occurs in (B1)). Equation (B1) includes already the possible noise increase due to water vapor, masked by $T_{m}$.

Now, for the usual low values of $T_{d s}$ (approximately $3 \mathrm{~K}$ or few Kelvins, if the antenna is not pointed to the sun or to another strong thermal-noise spot source), we can neglect the second term in (B1) and write

$$
T_{s k y} \approx \frac{10^{A / 10}-1}{10^{A / 10}} T_{m}<T_{m}
$$

Therefore, by assuming $T_{s k y}=T_{m}$ (worst case), we can write the inequality (2).

\section{APPENDIX C. AVERAGE EFFICIENCY CALCULATION}

The average efficiency (7) is given by the average value of the random variable $10^{-\frac{A}{10}}$, hence, in the long term, by the integral

$$
I=\int 10^{-\frac{y}{10}} f_{Y}(y) d y
$$

where $f_{Y}(y)$ is the density function of the random variable $y=A$. We can express this average as a function of the conditional complementary probability distribution $P_{c}(y)=\int_{y}^{\infty} f_{Y}(\zeta) d \zeta$, with $\zeta$ a dummy variable.

Because $f_{Y}(y)$ is the derivative of the distribution function $F_{Y}(y)=\int_{0}^{y} f_{Y}(\zeta) d \zeta$ and $P_{Y}(y)=1-F_{Y}(y)$, we can write

$$
I=\int 10^{-\frac{y}{10}} F_{Y}^{\prime}(y) d y
$$

Integrating (C2) by parts,

$$
\begin{aligned}
& I=\int_{0}^{\infty} 10^{-\frac{y}{10}} F_{Y}^{\prime}(y) d y= \\
& I=\left[F_{Y}(y) \times 10^{-\frac{y}{10}}\right]_{0}^{\infty}-\int_{0}^{\infty} 10^{-\frac{y}{10}}\left(-\frac{\ln 10}{10}\right) F_{Y}(y) d y= \\
& I=\frac{\ln 10}{10} \int_{0}^{\infty} 10^{-\frac{y}{10}} F_{Y}(y) d y= \\
& I=\frac{\ln 10}{10} \int_{0}^{\infty} 10^{-\frac{y}{10}}\left[1-P_{c}(y)\right] d y= \\
& I=\frac{\ln 10}{10} \times \frac{10}{\ln 10}-\frac{\ln 10}{10} \int_{0}^{\infty} 10^{-\frac{y}{10}} P_{c}(y) d y= \\
& I=1-\frac{\ln 10}{10} \int_{0}^{\infty} 10^{-\frac{y}{10}} P_{c}(y) d y
\end{aligned}
$$

(C3) gives (9). 


\section{APPENDIX D. AVERAGE EFFICIENCY BOUNDS}

The average efficiency is a random variable limited in a range. Any time we have integrals, we can use the Cauchy-Schwarz inequality for determining bounds. Let us calculate the upper limit. For real functions $f(t)$ and $g(t)$, it states that

$$
\left[\int f(t) g(t) d t\right]^{2} \leq \int f^{2}(t) d t \int g^{2}(t) d t
$$

In (D1), equality holds if $g(t)=\varepsilon f(t)$, with $\varepsilon=$ constant. By setting $f(t)=1$ and $g(t)=10^{-\frac{A(t)}{10}}$, we can write

$$
\left[\int_{T_{A}} 10^{-\frac{A(t)}{10}} \times 1 \times d t\right]^{2} \leq \int_{T_{A}} d t \int_{T_{A}} 10^{-\frac{A(t)}{5}} d t=T_{A} \int_{T_{A}} 10^{-\frac{A(t)}{5}} d t
$$

with equality sign holding when $10^{-\frac{A(t)}{10}}=\varepsilon$, that is, $A(t)=$ constant. Now, from this expression, we obtain

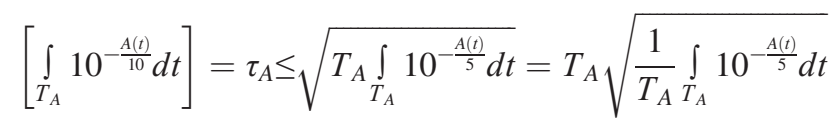

Hence, the upper bound

$$
\frac{\tau_{A}}{T_{A}}=\eta \leq \sqrt{\frac{1}{T_{A}} \int_{T_{A}} 10^{-\frac{A(t)}{5}} d t}=\eta_{\max }
$$

Therefore, according to calculations similar to those reported in Appendix C, $\eta_{\max }$ is given by

$$
\eta_{\max }=\sqrt{1-\frac{\ln 10}{5} \int_{0}^{\infty} 10^{-\frac{A}{5}} P(A) d A}
$$

Let us calculate the lower limit, hence the most conservative value. According to the Cauchy-Schwarz inequality, we can write

$$
\int_{T_{A}}\left[\sqrt{10^{-\frac{A(t)}{10}}}\right]^{2} d t \int_{T_{A}} d t \geq\left[\int_{T_{A}} \sqrt{10^{-\frac{A(t)}{10}}} d t\right]^{2}
$$

Hence,

$$
\int_{T_{A}} 10^{-\frac{A(t)}{10}} d t \times T_{A} \geq\left[\int_{T_{A}} 10^{-\frac{A(t)}{20}} d t\right]^{2}
$$

By dividing both members by $T_{A}^{2}$, we obtain

$$
\frac{1}{T_{A}} \int_{T_{A}} 10^{-\frac{A(t)}{10}} d t=\frac{\tau_{A}}{T_{A}}=\eta \geq\left[\frac{1}{T_{A}} \int_{T_{A}} 10^{-\frac{A(t)}{20}} d t\right]^{2}=\eta_{\min }
$$

which, integrated, gives

$$
\eta_{\min }=\left[1-\frac{\ln 10}{20} \int_{0}^{\infty} 10^{-\frac{A}{20}} P(A) d A\right]^{2}
$$

(D2) and (D3) set the range (10).

\section{REFERENCES}

1. Goldsmith AJ, Chua S-G. Adaptive coded modulation for fading channels. IEEE Trans Comm 1998; 46:595-602.

2. Panagopoulos AD, Arapoglou PM, Cottis PG. Satellite communications at Ku, Ka and V bands, propagation impairments and mitigation techniques. IEEE Commun Surveys Tut 2004, 3rd Quarter: 6:1-13.

3. Alberty E, Defever S, Moreau C, De Gaudenzi R, Ginesi A, Rinaldo R, Gallinaro G, Vernucci A. Adaptive coding and modulation for the DVB-S2 standard interactive applications: capacity assessment and key system issues. IEEE Wireless Commun 2007; 6:61-69. 
4. Bischl H, Brandt H, de Cola T, De Gaudenzi R, Eberlein E, Girault N, Alberty E, Lipp S, Rinaldo R, Rislow B, Skard JA, Tousch J, Ulbricht G. Adaptive coding and modulation for satellite broadband networks: from theory to practice. Int $J$ Satellite Comm Network 2010; 28:59-111.

5. Bauer R. Ka-band propagation measurements: an opportunity with the advanced communications technology satellite (ACTS). Proc IEEE 1997; 85:853-862.

6. Matricciani E. Physical-mathematical model of the dynamics of rain attenuation based on rain rate time series and two layer vertical structure of precipitation. Radio Sci 1996; 31:281-295.

7. Matricciani E, Riva C. The search for the most reliable long-term rain attenuation CDF of a slant path and the impact on prediction models. IEEE Trans Antenn Propag 2005; 53:3075-3079.

8. Siles GA, Riera JM, García del Pino P. Atmospheric attenuation in wireless communication systems at millimeter and $\mathrm{THz}$ frequencies. IEEE Antenn Propag Mag 2015; 57:48-61.

9. Matricciani E. Orbital diversity in resource-shared satellite communication systems above $10 \mathrm{GHz}$. IEEE J Sel Area Comm 1987; 5:714-723.

10. Marzano FS. Modeling antenna noise temperature due to rain clouds at microwave and millimeter-wave frequencies. IEEE Trans Antenn Propag 2006; 54:1305-1317.

\section{AUTHOR'S BIOGRAPHY}

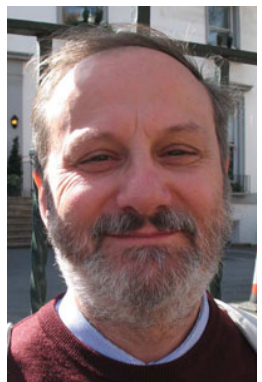

Emilio Matricciani was born in Italy in 1952. After serving in the Italian Army, he received the Laurea degree in Electronics Engineering at the Politecnico di Milano, Italy, in 1978. He joined the Politecnico di Milano in 1978 as recipient of a research scholarship, and in 1981, he became assistant professor of electrical communications. In 1987, he joined Università di Padova, Italy, as associate professor of microwaves. In 2001, he qualified as full professor of telecommunications. Since 1991, he works with Politecnico di Milano as professor of telecommunications. His research interests include satellite communications for fixed and mobile systems, deep-space communications, radio propagation at millimeter waves, rain effects on satellite system design, history of science, technology, and telecommunication. Most of his early experimental and theoretical activities have been carried out in the framework of propagation and communication experiments devised in the 1970s, 1980s, and 2000s at Politecnico di Milano by Francesco Carassa and Aldo Paraboni (satellites Sirio, ITALSAT, and Alphasat Aldo Paraboni experiment). In the 1990s and in the 2000s, he has conducted extensive research on communications with mobile terminals running in the rain and linked to satellites in the geostationary orbits, or in lower orbits, and on developing rain-attenuation prediction models useful to predict first-order (probability distributions) and second-order (fade durations, rates of change, and unavailability during the time of the day) statistics for satellite systems design. In addition to the institutional activities, such as lectures on satellite and terrestrial radio relays, he teaches scientific writing to $\mathrm{PhD}$ students and to graduate students. 